\title{
Determining the Dust Extinction of Gamma-ray Burst Host Galaxies: A Direct Method Based on Optical and X-ray Photometry
}

\author{
Y. $\mathrm{Li}^{*}$, Aigen $\mathrm{Li}^{\dagger}$ and D.M. Wei**** \\ *Purple Mountain Observatory, Chinese Academy of Sciences, Nanjing 210008, China \\ Department of Physics and Astronomy, University of Missouri, Columbia, MO 65211 \\ ${ }^{* *}$ Purple Mountain Observatory, Chinese Academy of Sciences, Nanjing 210008, China; Joint Center for \\ Particle Nuclear Physics and Cosmology of Purple Mountain Observatory - Nanjing University, Nanjing \\ 210008, China
}

\begin{abstract}
We propose a straightforward method to determine the extinction of GRB host galaxies by comparing the observed optical spectra to the intrinsic ones extrapolated from the X-ray spectra.The derived extinction curves are gray and do not resemble any extinction curves of local galaxies. The amount of extinction is rather large. We model the derived extinction curves in terms of the silicate-graphite interstellar grain model and obtain the dust size distribution skewed to large grains. We determine, for the first time, the local dust-to-gas ratios of GRB host galaxies using the model-derived dust parameters and the hydrogen column densities determined from X-ray absorptions.
\end{abstract}

Keywords: Gamma-ray: bursts

PACS: $98.70 . \mathrm{Rz}$

\section{INTRODUCTION}

It is widely acknowledged that the long-duration gamma-ray bursts (GRB) are associated with the collapse of massive stars [40]. In this scenario, GRBs are born and explode inside dense, dusty environments. To study the dust and gas properties in the vicinity of GRBs can help (1) to understand the interstellar medium (ISM) of star-forming galaxies throughout cosmic history, (2) to correct for the extinction of optical emission in GRB afterglow analysis and (3) to reveal the nature of so-called "dark bursts"[14].

The dust extinction of GRB host galaxies is traditionally modeled using either the Milky Way (MW), the Large Magellanic Cloud (LMC), the Small Magellanic Cloud (SMC), or other presumed extinction curves[3] [32, 13, 28, 31, 35]. Chen et al. (2006)[6] made the first effort to determine the extinction curves for GRB host galaxies without a priori assumption of the extinction law. The derived extinction curves differ from any known extinction laws of the Milky Way and external galaxies, challenging the traditional method commonly used in determining the extinction curves of GRB host galaxies.

Here we propose a novel, straightforward method to determine the extinction of GRB host galaxies by comparing the observed optical spectra to the intrinsic ones extrapolated from the X-ray spectra based on the standard fireball model.

\section{METHOD}

The standard fireball model [24] predicts that the afterglow emission is produced by synchrotron radiation of electrons accelerated by the forward shock. In this model, with typical parameters, the optical to X-ray spectra can be described by a broken power law with indices $\beta=(p-1) / 2$ for $v<v_{c}$ or $\beta=p / 2$ for $v>v_{c}$, where $v_{c}$ is the cooling frequency and $p$ is the electron energy distribution index. In most cases, the cooling break position is hard to determine. If the decay indices $\alpha$ of X-ray and optical bands are different, the cooling frequency lies between them, making it difficult to calculate the intrinsic optical flux from X-ray data. However, if the decay indices $\alpha$ of X-ray and optical bands are the same, then the optical and X-ray should lie on the same spectral segment, rendering it possible to calculate the intrinsic flux density in any optical band from $F_{\lambda}=F_{X}\left(\lambda / \lambda_{X}\right)^{\beta-2}$, where $\beta$ is the X-ray afterglow spectral index that 
we get from fitting the X-ray spectrum, and $F_{X}$ is the X-ray flux density. After corrected for the Galactic extinction using the reddening maps of Schlegel et al. (1998)[29], the observed spectral energy distribution (SED) of GRB at redshift $z$ can be described as $F_{\lambda(1+z)}=F_{\lambda} \exp \left(-A_{\lambda} / 1.086\right)$. Therefore, the extinction of the GRB host galaxy can be given by

$$
A_{\lambda}=1.086 \ln \frac{F_{X}\left(\lambda / \lambda_{X}\right)^{\beta-2}}{F_{\lambda(1+z)}} .
$$

With $A_{V}$ interpolated, we can obtain the extinction curves (normalized to $V$ band) of the GRB host galaxies.

We then fit the derived extinction curve $A_{\lambda} / A_{V}$ with the standard silicate-graphite interstellar dust model.The grain size distribution for both silicate and graphite is modeled with: $d n=N(a) d a \sim a^{-\eta} \exp \left(-a / a_{c}\right) d a$, where $a$ is the grain radius (assumed spherical), ranging from $a_{\min }=0.005 \mu \mathrm{m}$ to $a_{\max }=2.5 \mu \mathrm{m}, a_{c}$ is the cut-off size.

With the fitted dust parameters, we can estimate the dust-to-gas ratio in each of the GRB host galaxies:

$$
\frac{m_{\mathrm{dust}}}{m_{\mathrm{gas}}}=\frac{M_{\mathrm{gra}}+M_{\mathrm{sil}}}{1.4 N_{\mathrm{H}} \mu_{\mathrm{H}}}
$$

where $N_{\mathrm{H}}$ is the hydrogen column density in the host galaxy. The dust column density $N_{d}$ can be derived from

$$
A_{\lambda}=1.086 \int_{a_{\min }}^{a_{\max }} N(a) \pi a^{2}\left[f_{\text {gra }} Q_{\text {ext,gra }}(a, \lambda)+\left(1-f_{\text {gra }}\right) Q_{\text {ext,sil }}(a, \lambda)\right] d a N_{d},
$$

where $Q_{\text {ext,gra }}(a, \lambda)$ and $Q_{\text {ext,sil }}(a, \lambda)$ is the extinction efficiency of dust of radius $a$ at wavelength $\lambda$ for graphite and silicate material, respectively.

\section{DATA AND RESULTS}

We select four GRBs that have both optical and X-ray observations. Photometric data are taken from literature (see Table 1).For GRB 020405, GRB 030227 and GRB 060729, we adopt the spectra obtained when the cooling frequency $v_{c}$ falls below the optical band, indicating an intrinsic single power law spectrum through optical and X-ray bands as discussed above. For GRB 061126, the decay indices of X-ray and optical bands are different, indicating a break frequency lying between them. At $\sim 30 \mathrm{ks}$, the $\mathrm{R}$ band afterglow shows a break, which can be interpreted as the spectral break passing through the $\mathrm{R}$ band, ${ }^{1}$ allowing us to calculate the intrinsic optical flux from $F_{v} / F_{X}=\left(v / v_{c}\right)^{-\beta+0.5}\left(v_{c} / v_{X}\right)^{-\beta}$.

We present in Table 2 the $V$-band extinction versus hydrogen column density $\left(A_{V} / N_{\mathrm{H}}\right)$ and the dust-to-gas ratio. The derived extinction curves of the four bursts are rather "gray" (see Fig. 2). Since all these extinction curves have very similar slope, we put all the extinction data of the four bursts together to fit them to the silicate-graphite grain model. The best fit parameters are $\eta \approx 2.60, a_{c} \approx 2.0 \mu \mathrm{m}$, and $f_{\text {gra }} \approx 0$. A prominent feature is the considerably small $\eta$ (the canonical value of $\eta$ is 3.5), indicating a grain size distribution skewed towards substantially large grains.

\section{DISCUSSION}

The "collapsar" model predicts GRBs to occur in active star-forming regions similar to Galactic molecular clouds [11] which are heavily enshrouded by dust [37,36]. Observations supporting the existence of large amount of dust include the emission and absorption features in some X-ray afterglows [1,22], large column densities of heavy elements revealed by optical spectroscopy studies [26,27], and the non-detection at optical wavelengths for more than half the well-localized GRBs [10]. In contradiction with these evidence, traditional SED fitting often finds small extinction, primarily because the best fit model in most cases is the SMC-type extinction which, with a steep rise into the far ultraviolet (UV), often requires a small $A_{V}$ to fit the spectrum $[13,28,35]$. Our work, showing considerably large $A_{V}$ compared to that fitted with traditional method, is more consistent with theoretical prediction and observations. In

\footnotetext{
${ }^{1}$ We note, however, that this burst, like many other Swift bursts, does not obey the closure relation in the standard afterglow model [21], which adds uncertainties to our analysis. But the uncertainty of break frequency does not appear to affect the shape of the derived extinction curve - as can be seen in Figure 2, the extinction curve for the other three bursts remains gray even if we ignore the GRB 061126 data.
} 


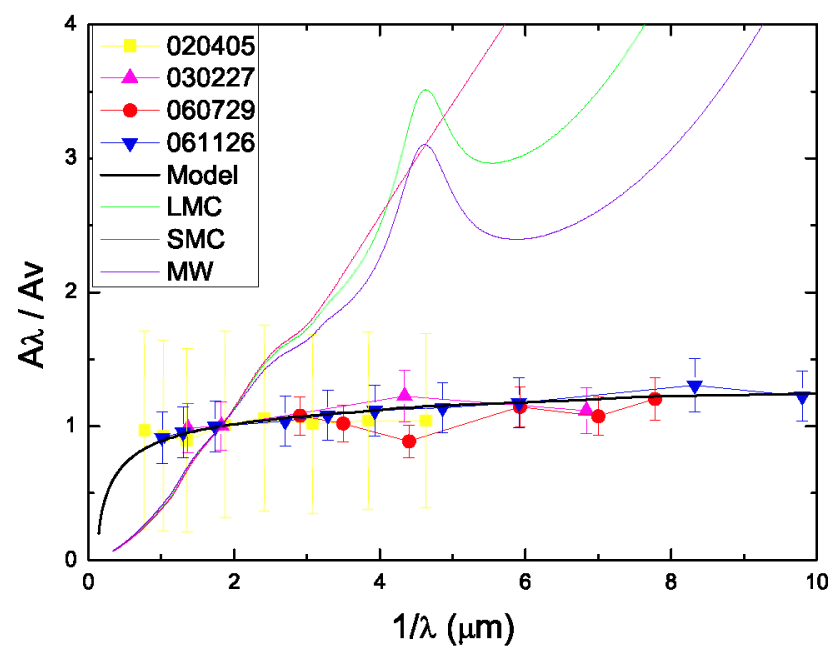

FIGURE 1. Rest-frame extinction curves (normalized to the $V$ band) for 4 GRB host galaxies and the best-fit extinction curve from the silicategraphite model (thick black line). Also shown are the Milky Way, SMC and LMC extinction curves.

TABLE 1. Observational Properties of the four GRBs

\begin{tabular}{ccccccccc}
\hline GRB & $z$ & $\beta$ & $F_{X}(\mu \mathrm{Jy})$ & $t$ (days) & $T_{90}(\mathrm{~s})$ & $\alpha_{o}$ & $\alpha_{X}$ & References \\
\hline 020405 & 0.691 & $1.0 \pm 0.2$ & 0.23 & 1.98 & 60 & $1.54 \pm 0.06$ & $1.97 \pm 1.10$ & {$[2,33]$} \\
030227 & 4 & $0.94 \pm 0.05$ & 0.125 & 0.87 & 18 & $0.95 \pm 0.16$ & $0.97 \pm 0.07$ & {$[5,19]$} \\
060729 & 0.54 & $1.06 \pm 0.01$ & 0.2 & 4.6 & 115 & $1.27 \pm 0.10$ & $1.29 \pm 0.03$ & {$[7]$} \\
061126 & 1.1588 & $0.5 \pm 0.07$ & 40.5 & 0.023 & 191 & $0.75 \pm 0.06$ & $1.31 \pm 0.01$ & {$[21]$} \\
\hline $06072 *^{* *}$ & 0.54 & $1.06 \pm 0.01$ & 3.46 & 0.35 & 115 & $0.26 \pm 0.07$ & $0.35 \pm 0.15$ & {$[7]$} \\
\hline
\end{tabular}

* Data are taken during the plateau phase.

addition, Rol et al. (2007) [23]found that for GRB 051022 a lower limit of $A_{V} \approx 4.4 \mathrm{Mag}$ was needed, which implies that at least in some GRBs the extinction $A_{V}$ is rather large.

The extinction curve derived in our work is flat, almost independent of wavelength. This result is in good agreement with other works fitting the SEDs of these bursts $[33,16]$. In particular, Perley et al. (2007) found that for GRB 061126 the extinction curve is gray. Gray extinction has also been observed in Galactic dense clouds [4] and in the circumnuclear region of some AGNs (see [15] for a review). Gray extinction is produced by a dust distribution biased towards large grains, which may form from (1) grain coagulation naturally expected in the dense circumstance near GRBs $[17,18],(2)$ the biased evaporation of smaller grains due to the intense X-ray and UV radiation up to $\sim 20$ parsecs from the GRB $[39,8,25]$, and (3) preferential destruction of small grains by high energy ions in fast shocks [12]. Perna et al. (2003)[20] computed the extinction curve that is obtained if standard Galactic dust is exposed to a GRB lasting more than a few tens of secondsand found that the extinction curve can be very flat, chiming with our result.

In accordance with previous works, we find that the average $A_{V} / N_{\mathrm{H}}$ is smaller than that in the Milky Way, which is usually ascribed to a lower dust-to-gas ratio in GRB vicinities [38]. However, there is no obvious reason why the amount of dust is low in the dense environment surrounding GRBs. We note that the dust extinction is very sensitive to the dust size distribution, for larger grains the extinction (on a per unit mass basis) is low, but the amount of dust may be still high [16]. In fact, based on the model fit dust parameters, the dust-to-gas ratios for most bursts are larger than that in the Milky Way. On the other hand, grain growth through coagulation in dense molecular clouds enshrouding GRBs is expected and this would result in a dust size distribution biased in favour of large grains, a flat extinction curve, and a reduced $A_{V} / N_{\mathrm{H}}$. 
TABLE 2. $V$-band extinction and dust-to-gas ratio in the rest frame of each burst.

\begin{tabular}{ccccc}
\hline GRB & $A_{V}(\mathrm{mag})$ & $N_{\mathrm{H}}\left(10^{22} \mathrm{~cm}^{-2}\right)$ & $A_{V} / N_{\mathrm{H}}\left(10^{-22} \mathrm{Magcm}^{2}\right)$ & $m_{\text {dust }} / m_{\text {gas }}\left(10^{-2}\right)$ \\
\hline 020405 & $2.50 \pm 1.17$ & $0.8 \pm 0.2$ & $3.2 \pm 1.6$ & $0.99 \pm 0.52$ \\
030227 & $2.57 \pm 0.32$ & $6.8_{-3.8}^{+1.8}$ & $0.38_{-0.11}^{+0.22}$ & $0.12_{-0.03}^{+0.07}$ \\
060729 & $1.59 \pm 0.20$ & $0.076 \pm 0.003$ & $20.9 \pm 2.8$ & $6.5 \pm 0.9$ \\
061126 & $3.35 \pm 0.43$ & $1.1 \pm 0.3$ & $3.0 \pm 0.9$ & $0.94 \pm 0.28$ \\
\hline $060729^{*}$ & $1.70 \pm 0.20$ & $0.076 \pm 0.003$ & $22.4 \pm 2.8$ & $6.9 \pm 0.9$ \\
\hline
\end{tabular}

\section{ACKNOWLEDGMENTS}

We thank S.L. Chen, S.L. Liang and Y.Z. Fan for helpful discussion. D.M.W. is supported by the NSFC grants 10621303 and 10673034, and the National Basic Research Program of China (973 Program 2007CB815404). A.L. is supported in part by a NASA/Swift Theory Program, a NASA/Chandra Theory Program, and the NSFC Outstanding Oversea Young Scholarship.

\section{REFERENCES}

1. Antonelli L.A., et al. 2000, ApJ, 545, L39

2. Berger, E. J., Soderberg, A. M., Frail, D. A., \& Kulkarni, S. R. 2003, ApJ, 587, L5

3. Calzetti, D., Kinney, A. L., \& Storchi-Bergmann, T. 1994, ApJ, 429, 582

4. Cardelli, J. A., Clayton, G. C., \& Mathis, J. S. 1988, ApJ, 329, L33

5. Castro-Tirado, A., et al. 2003, A\&A, 411, L315

6. Chen, S.L., Li, A., \& Wei, D.M. 2006, ApJ, 647, L13

7. Dirk Grupe, et al. 2007, ApJ, 662, 443

8. Fruchter, A., Krolik, J. H., \& Rhoads, J. E. 2001, ApJ, 563, 597

9. Fynbo J.P.U. et al. 2006, A\&A, 451, L47

10. Jakobsson, P., et al. 2004, ApJ 617, L21

11. Jakobsson, P., et al. 2006, A\&A, 460, L13

12. Jones A. P. 2004, in Astrophysics of Dust, eds. A.N. Witt, G.C. Clayton, \& B.T. Draine, ASP Conf. Ser., 309,347

13. Kann, D. A., Klose, S., \& Zeh, A. 2006, ApJ, 641, 993

14. Lazzati, D., Covino, S., \& Ghisellini, G. 2002, MNRAS, 330, 583

15. Li, A. 2007, in The Central Engine of Active Galactic Nuclei, eds. L.C. Ho, \& J.-M. Wang, ASP Conf. Ser., 373,561

16. Li, A., Liang, S.L., Kann, D.A., Klose, S., \& Wei, D.M. 2007, in preparation

17. Maiolino, R., et al. 2001a, A\&A, 365, 28

18. Maiolino, R., Marconi, A., \& Oliva, E. 2001b, A\&A, 365, 37

19. Mereghetti, S., et al. 2003, ApJ, 590, L73

20. Perna, R., Lazzati, D., \& Fiore, F. 2003, ApJ, 585, 775

21. Perley, D.A., et al. 2007, ApJ, in press

22. Piro, L., et al. 1999, ApJ, 514, L73

23. Rol, E., et al. 2007, ApJ, in press

24. Sari, R., Piran, T., \& Narayan, R. 1998, ApJ, 497, L17

25. Savaglio, S., Fall, S.M., \& Fiore, F. 2003, ApJ, 585, 638

26. Savaglio, S., \& Fall, S.M. 2004, ApJ, 614, 293

27. Savaglio, S. 2006, New Journal of Phys., 8, 195

28. Schady, P., et al. 2007, MNRAS, 377, 273

29. Schlegel, D.J., Finkbeiner, D. P., \& Davis, M. 1998, ApJ, 500, 525

30. Stanek, K.Z., et al. 2006, Acta Astronomica, 56, 333

31. Starling, R.L.C., et al. 2007, ApJ, 661, 787

32. Stratta, G., Fiore, F., Antonelli, L. A., Piro, L., \& De Pasquale, M. 2004, ApJ, 608, 846

33. Stratta, G., Perna, R., Lazzati, D., Fiore, F., Antonelli, L. A., \& Conciatore, M. L. 2005, aap, 441, 83

34. Stratta, G., Maiolino, R., Fiore, F., \& D'Elia, V. 2007, ApJ, 661, L9

35. Tagliaferri, G., et al. 2007, astro-ph/0701591

36. Tanvir, N.R., et al. 2004, MNRAS, 352, 1073

37. Trentham, N., Ramirez-Ruiz, E., \& Blain, A.W. 2002, MNRAS, 334, 983

38. Watson, D., et al. 2006, ApJ, 652, 1011

39. Waxman, E., \& Draine, B.T. 2000, ApJ, 537, 796 2001, ApJ, 548, 296

40. Woosley, S.E. 1993, ApJ, 405, 273 